\title{
FUNDAMENTOS DEL TRABAJO CON EL MAPA COMO MÉTODO EN EL PROCESO DE ENSEÑANZA-APRENDIZAJE DE LA GEOGRAFÍA
}

\author{
Josbel Gómez Torres ${ }^{1}$
}

Universidad de Artemisa

Pedro Pablo Recio Molina ${ }^{2}$

Universidad de Ciencias Pedagógicas Enrique José Varona

Elio Lázaro Amador Lorenzo ${ }^{3}$

Universidad de Ciencias Agrícolas de La Habana

Recibido 02/05/2019 Aceptado 25/11/2019 El siguiente artículo tiene el propósito de revelar el resultado
de un estudio crítico referente al trabajo con el mapa como mé-
todo en el proceso de enseñanza-aprendizaje de la Geografía
en Cuba y las concepciones generales acerca de su utilización
desde una profunda revisión bibliográfica y otros métodos de
investigación.

$\because \quad$ The following article has the purpose of revealing the result of a critical study regarding the work with the map as a method in the teaching-learning process of Geography in Cuba and the general conceptions about its use from a deep bibliographical revision and others Research Methods.

DOI

https://doi.org/10.15366/didacticas2019.21.001

PALABRAS CLAVE

Enseñanza-Aprendizaje; Geografía; Trabajo con el mapa; Método de Enseñanza-Aprendizaje.

KEYWORDS

1. josbelg@uart.edu.cu Teaching-Learning; Geography; Working with the map; TeachingLearning Method. 


\section{INTRODUCCIÓN}

Desde hace mucho tiempo el mapa geográfico se ha definido como una representación reducida de la superficie terrestre en un plano. Sin embargo, esta definición es válida tanto para una fotografía cualquiera de la superficie terrestre como para un paisaje o representación artística de una localidad. Por consiguiente, debe ser completada señalando aquellas propiedades esenciales que diferencian al mapa de otras representaciones de la superficie terrestre, dándole sus características especiales.

El contenido del mapa no se limita a una representación de la superficie terrestre; mientras que los mapas modernos pueden mostrar los más diversos fenómenos naturales y económico-sociales; por ejemplo, la distribución de la temperatura, la presión del aire, la composición de la población por nacionalidades, etc., siempre que sean representados en relación con la superficie terrestre.

En términos generales, podemos decir que el mapa geográfico es el material cartográfico, impreso o digital, que representa de forma reducida, generalizada y matemáticamente determinada la distribución, el estado y los vínculos de los objetos, fenómenos y procesos que se dan en la superficie terrestre sobre un plano; empleando para ello, distintas proyecciones cartográficas, el uso de escalas y la escritura convencional de símbolos propios, formas y colores. Todo ello con la combinación más eficiente de los sistemas de información geográfica, la teledetección, la fotogrametría y métodos de representación cartográfica, que varían de acuerdo a la asignación concreta y nivel de generalización que se quiere representar a diferentes escalas planetarias (Recio Molina, Gómez Torres, Díaz Soto, Feria Ávila \& Cáseres, 2018, 23).

Es importante no sólo tener un buen mapa, sino poder trabajar sobre él, para obtener todo lo que pueda ofrecernos; de otra manera quedaría en un grado considerable como una cosa en sí.

Entre la diversidad de líneas para el uso práctico y científico del mapa se pueden destacar los aspectos siguientes:

- El estudio en los mapas de las localidades y, en general, de las representaciones de los fenómenos y objetos con sus propiedades y particularidades (lectura del mapa).

○ La orientación por el mapa, es decir, el empleo del mapa como guía, en una localidad.

○ Los trabajos cartométricos, o sea, la determinación en los mapas de los objetos por medio de sus características espaciales cuantitativas.

- La obtención de nuevas características de los fenómenos (objetos) y el conocimiento de los resultados de la elaboración (reconstrucción) de los mapas geográficos. 
○ El empleo de los mapas para el análisis y el conocimiento de los fenómenos incluidos en sus objetivos:

a) La aclaración de las leyes de la distribución espacial de los fenómenos.

b) El estudio de las correlaciones y dependencia entre los fenómenos.

c) El estudio del desarrollo de los fenómenos.

d) El pronóstico de la extensión y el desarrollo de los fenómenos.

Para plantear correctamente los fundamentos de los conocimientos cartográficos, inculcar en los educandos los hábitos del trabajo con los mapas, aficionar a los educandos en su lectura y estudio, el maestro de Geografía debe orientarse con suficiente libertad en la Cartografía, conocer todas sus ventajas y problemas, y fundamentalmente, presentar de forma evidente, el papel de la Cartografía en la solución de los problemas económicos, en la difusión de los conocimientos y la cultura y la vida de la sociedad moderna.

En estas condiciones es necesario perfeccionar la impartición de los temas cartográficos en la escuela. Es necesario, nuevamente, examinar la importancia y el lugar del trabajo con el mapa en la Geografía y otras ciencias que estudian la Tierra y la sociedad, subrayando, que la Cartografía moderna es, ante todo, una ciencia cognoscitiva.

Muchos son los autores que han obtenido resultados significativos en cuanto al desarrollo del trabajo con el mapa: Barraqué, G. (1991), Pérez, M. (1991), Báyeux, F. (1999), Gutiérrez, A. (1999), Feria, H. (2004), Lagunas, J. (2005), Recio, P. (2006), Jérez, G. (2011), Durán, D. (2009), Araya P. (2014), Gómez (2016).

Estos autores indistintamente aportan conceptos, acciones, ideas rectoras, concepciones pedagógicas, metodologías, estrategias y alternativas para desarrollar el trabajo con el mapa, pero no se encontró evidencias de investigaciones que reflejaran los fundamentos del trabajo con el mapa como método.

Como parte de los resultados del proyecto "Educación Geográfica para el Desarrollo Sostenible", realizado durante los años 2016 hasta 2019 en la Universidad de Ciencias Pedagógica "Enrique José Varona”, los autores identificaron carencias en cuanto al trabajo con el mapa en el proceso de enseñanza-aprendizaje de la Geografía y su utilización como método de enseñanza. Todo ello posibilitó precisar cómo situación problemática:

- En investigaciones relacionadas con las Ciencias Naturales en general y con la Geografía en particular, no se identificaron resultados científicos en cuanto a la utilización del trabajo con el mapa como método en el proceso de enseñanza-aprendizaje de la Geografía.

- Se observa en los programas de Ciencias Naturales y Geografía del nivel educativo Secundaria Básica, una orientación del trabajo con el mapa; sin embargo, este último no se contextualiza como método en el proceso de enseñanza- prendizaje de la Geografía en los educandos de esta educación. 
- De la experiencia como profesor de Geografía se identifica que es insuficiente la orientación que realizan los profesores a sus educandos para el desarrollo del trabajo con el mapa en clases.

- El nivel de desarrollo de las habilidades de trabajo con el mapa en los educandos de Educación Secundaria Básica es deficiente.

Atendiendo a los elementos anteriores, los autores de esta investigación determinaron resolver la siguiente pregunta de investigación: ¿Qué fundamentos teóricos y metodológicos sustentan el trabajo con el mapa como método en el proceso de enseñanza-aprendizaje de la Geografía?

El siguiente artículo tiene el propósito de revelar el resultado de un estudio crítico referente al trabajo con el mapa como método en el proceso de enseñanza-aprendizaje de la Geografía en Cuba y las concepciones generales acerca de su utilización.

\section{DESARROLLO}

Para el desarrollo de la investigación se utilizaron diferentes métodos y técnicas de investigación. Se utilizó el Método Histórico-Lógico para conocer los antecedentes y tendencias actuales, tanto nacionales como internacionales, sobre del trabajo con el mapa, lo que permitió identificar las primeras aproximaciones al comportamiento del estudio crítico.

La Sistematización permitió organizar los conocimientos a partir del comportamiento de la práctica educativa y la literatura consultada, para establecer relaciones del trabajo con el mapa como método para su desarrollo en el proceso de enseñanza-aprendizaje de la Geografía en el nivel educativo Secundaria Básica, lo que favoreció su orientación didáctica-metodológica.

De igual manera se realizó un análisis crítico de las variadas fuentes de información consultadas en el transcurso de la investigación, referidas al trabajo con el mapa en el proceso de enseñanza-aprendizaje de la Geografía en el nivel educativo Secundaria Básica; la búsqueda de elementos que fundamenten la concepción didáctica elaborada, así como para las reflexiones en torno a las acciones desarrolladas durante el diseño, desarrollo y evaluación de la propuesta. Igualmente se aplicó en el procesamiento de información de los instrumentos durante la investigación.

Se revisaron documentos normativos, resoluciones, programas de asignaturas, lo que proporcionó la información necesaria sobre los referentes teórico-metodológicos que sobre el trabajo con el mapa en el proceso de enseñanza-aprendizaje de la Geografía en el nivel educativo Secundaria Básica se tenían. Se aplicó una encuesta a 112 educandos con la intención de buscar cuáles eran los conocimientos que poseían los educandos acerca la utilización del trabajo con el mapa y de las habilidades que presupone para el desarrollo de estas temáticas en el aula. 
Por último, una encuesta a 20 profesores en las Secundarias Básicas, con el propósito de constatar el trabajo con el mapa como método, cuáles son los temas tratados y la importancia que le brindan al mapa en la comprensión de los contenidos en el proceso de enseñanza-aprendizaje de la Geografía...

\section{El trabajo con el mapa en el proceso de enseñanza-aprendizaje de la Geografía. Concep- ciones generales acerca de su utilización en la Educación Secundaria Básica}

La Geografía, como disciplina docente, tiene una importancia extraordinaria porque forma en el estudiante convicciones, ideales, aptitudes y una cultura general que le permite el conocimiento, análisis y obtención de conclusiones sobre condiciones existentes en los diferentes estados y países del mundo y posibilita adoptar posiciones para llegar a explicar un mundo regido por la razón, la justicia y el amor entre los hombres y la naturaleza de manera armónica. De igual modo, contribuye a la formación integral de los educandos en su tránsito por los diferentes niveles educativos, dado que propicia una formación que amplía distintos puntos de vista y criterios educativos básicos, los que quedan identificados en el modelo de plan de estudio en Cuba en relación con: La concepción científica del mundo; el desarrollo de conocimientos, hábitos y habilidades en correspondencia con el desarrollo actual de las ciencias geográficas; la educación económica, ecológica y política; los cambios que ocurren en el mapa político del mundo y la desigual distribución de las riquezas; los problemas medioambientales y sus consecuencias, y la necesidad de un desarrollo sostenible; la educación moral, que a partir de los conocimientos geográficos contribuye a fortalecer el patriotismo, el internacionalismo, los sentimientos éticos y morales, así como el amor al trabajo; el sistema de habilidades relacionadas con los conocimientos geográficos, en particular los cartográficos, para que puedan ser utilizados en la vida práctica y como vía de apropiación de otros nuevos.

En Cuba, como resultado del perfeccionamiento continuo del Sistema Nacional de Educación (SNE), se han realizado transformaciones curriculares en las educaciones Primaria, Secundaria Básica y Preuniversitaria, a lo largo del decurso de este proceso en diferentes etapas.

Para caracterizar este proceso se asumen las periodizadas por Hernández Herrera (2005) en su Tesis Doctoral "La enseñanza de la Geografía de Cuba, un estudio histórico crítico desde 1959 hasta el año 2000" y los aportes de la Tesis de Doctorado de Recio Molina (2006) "Estrategia didáctica de trabajo con el mapa con enfoque desarrollador en el proceso de enseñanza-aprendizaje de la Geografía en la secundaria básica”.

En la primera etapa del perfeccionamiento del SNE (1975-1981), la estructura que asumió la Geografía escolar en la escuela cubana fue de desarrollo lineal. Los contenidos a desarrollar por los educandos se caracterizaron por tener un ordenamiento gradual, donde estos se presentan una sola vez a un nivel determinado y se van elevando gradualmente sin regresar a él, evitando repeticiones innecesarias. 
La enseñanza de la Geografía en Educación Secundaria Básica formaba parte del ciclo sistemático de los conocimientos geográficos, que comenzaba en la Geografía Física Elemental que se impartía en 5to grado y la Geografía Física de los Continentes I en el 6to grado de la enseñanza primaria.

En 7mo grado, el programa Geografía Física de los Continentes II daba continuidad al iniciado en el nivel anterior; en 8vo se enseñaba la Geografía Física de Cuba y el 9no grado, último de la Educación Secundaria Básica, culminaba con la asignatura Geografía Económica de Cuba.

En cuanto a los métodos de enseñanza-aprendizaje, se asumió la clasificación binaria de los métodos que tienen en cuenta la fuente de obtención de los conocimientos (verbales, visuales y prácticos), y los niveles de independencia cognoscitiva a alcanzar por el estudiante. Prevaleció la forma tradicional de manejo del mapa como medio, en la que solo se le exigía al estudiante la memorización de los accidentes y la reproducción verbal de estos. Se puede plantear que constituía un medio de enseñanza-aprendizaje indispensable para la enseñanza de la Geografía, pero no se concebía el trabajo con el mapa como una estructuración didáctica.

Durante el quinquenio 1981-1985, segunda etapa del perfeccionamiento del Sistema Nacional de Educación, se determinó estructurar un plan de estudio de la Geografía escolar escalonado y ascendente, centrado en el país natal, de manera que, progresivamente, los educandos fueran apropiándose de los conocimientos geográficos y de las habilidades intelectuales y prácticas correspondientes, sin que existieran repeticiones, sino que, por el contrario, fueran complementándose y ampliando los conceptos y las definiciones en los distintos grados.

Para la Educación Secundaria Básica como continuación del ciclo básico comenzado desde el 6to grado, el plan de estudio de Geografía se aplicaría, al igual que el de otras asignaturas, de manera escalonada; es decir, se impartiría primero el programa de $7 \mathrm{mo}$ grado con la Geografía Física de los Continentes, posteriormente se seguiría Geografía Física General y de Cuba y Geografía Económica General y de Cuba en 8vo grado.

En 9no grado se mantuvo el programa de Geografía Económica de Países, en tránsito desde la etapa anterior, el que sufrió adecuaciones sistemáticas desde el curso 1986-1987 hasta el curso 1990-1991 en que deja de impartirse y se sustituye por Temas de Geografía de Cuba.

Con respecto a los métodos de enseñanza-aprendizaje predominó, al igual que la etapa anterior, la clasificación binaria de los métodos que tienen en cuenta la fuente de conocimientos (verbales, visuales y prácticos), y los niveles de independencia cognoscitiva a alcanzar por el estudiante.

De igual manera, el mapa y su proceder continuaba siendo un medio indispensable para la enseñanza de la Geografía, pero aún no se concebía el trabajo con el mapa como una 
estructuración didáctica, aunque autores como Pérez Capote, (1991); Barraqué Nicolau, (1991); Caner Román, (1994); lo mencionaban o lo referían como método en sus textos.

La relación hombre-naturaleza estuvo presente con un enfoque sistémico y el estudio de la naturaleza se situó en el centro de los conocimientos geográficos a la par del estudio de la localidad.

Desde 1991 hasta 2000 comienzan, para la enseñanza de la Geografía, cambios en sus programas de estudio. A finales de la década de los ochenta tienen lugar en Eurasia algunos acontecimientos económicos y sociales que trajeron consigo cambios significativos en el espacio geográfico que conforma el mapa político del mundo actual. Esto impuso adecuaciones en los sistemas de conocimientos de la Geografía que se impartían en todos los niveles de educación.

Para la Educación Secundaria Básica se ofrecieron indicaciones específicas por el Ministerio de Educación a todos los profesores con vistas a la adecuación de algunos objetivos y al desarrollo de algunas temáticas. Así, deja de impartirse la asignatura Geografía de los Continentes durante el curso 1990-1991, por considerarse que primero deberían tratarse los aspectos físico-geográficos y los económico-geográficos para después integrarlos en la región o el continente; de igual manera, en el curso escolar 1990-1991, desaparece en 9no. grado el programa de Geografía Económica de Países y surge el extracurricular "Temas de Geografía de Cuba”, como vía para completar, ampliar y consolidar la formación del estudiante sobre el país natal.

Con respecto a los métodos prevaleció la clasificación según los niveles de independencia cognoscitiva a alcanzar por el estudiante, se concibe el trabajo con el mapa como habilidad básica de la enseñanza de la Geografía.

A partir del año 2001, con la puesta en práctica de un nuevo modelo para la Educación Secundaria Básica, ocurren transformaciones importantes en los programas de estudio. Para las asignaturas geográficas las transformaciones fueron ocurriendo paulatinamente, desde el año 2001 hasta el 2014.

Así, comienza a impartirse desde el 2001 hasta el 2003, Geografía 2 en 7mo grado, con un marcado enfoque geográfico; en 8vo grado se imparte Geografía 2, con un marcado enfoque regional, y en 9no Geografía de Cuba, con un conocimiento más completo de la Geografía del país.

A partir del 2003 hasta el 2010, la Geografía continúa experimentando cambios en el currículo de la Educación Secundaria Básica. Comienza a impartirse Ciencias Naturales para los grados $7 \mathrm{mo}$ y $8 \mathrm{vo}$ grados, donde se integran los contenidos físico-geográficos y económico-geográficos con los contenidos de Biología, Física y Química respectivamente. Para el 9no grado se mantiene la Geografía de Cuba.

Desde el 2010 hasta la actualidad se imparte Ciencias Naturales en 7mo grado, con contenidos de Geografía Física y Biología; en 8vo grado, Geografía 2 con contenidos de 
Geografía Económica y Social, y Geografía Regional y Geografía 3 (9no grado), abarca contenidos de Geografía de Cuba.

Con respecto a los métodos, prevalece la clasificación según los niveles de independencia cognoscitiva. El trabajo con el mapa se concibe como una concepción didáctica con un enfoque desarrollador en función de tres niveles de lectura del mapa: reproducción, interpretación y extrapolación.

De esta manera queda conformado, como en años anteriores, un plan de estudio de la Geografía de forma lineal, escalonada y centrada en el país natal, donde el trabajo con el mapa es objetivo en los programas de Geografía, constituye una exigencia de esta y es, a la vez, un eje central del aprendizaje de los contenidos de sus asignaturas, según se muestra en la figura siguiente.

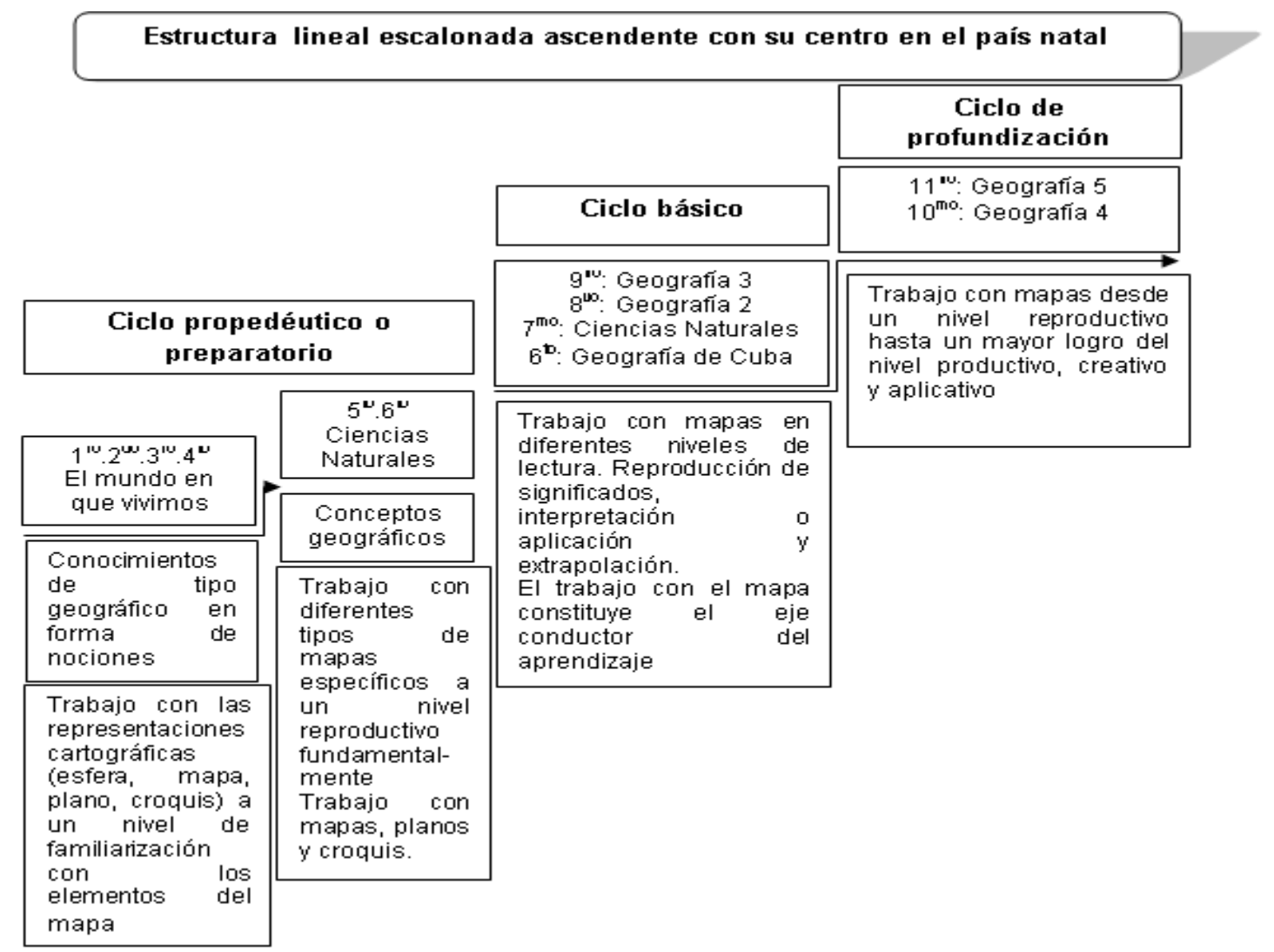

Figura 1. Plan de estudio de la Geografía (2010-2018) y su relación con el desarrollo del trabajo con el mapa. Fuente: Tomada de Hernández, H (2005) y adaptada por Gómez J. (2014)

En los programas vigentes en la Educación Secundaria Básica se plantea, para las asignaturas geográficas, exigencias básicas y entre ellas aparecen:

○ El trabajo con mapas en diferentes niveles: reproducción, interpretación y extrapolación. 
- Ubicación espacial de hechos, fenómenos y procesos históricos que han ocurrido y ocurren en el mundo, en particular en Cuba.

○ Utilización de algoritmos de trabajo (léase planes tipos) para el estudio de los paisajes, países y regiones, y dentro de estos, situación geográfica del objeto de estudio.

Estas exigencias dejan ver a las claras que el trabajo con el mapa es para la Geografía un elemento consustancial a su objeto de estudio; por lo tanto, constituye un objetivo de los programas escolares, por lo que no se concibe una clase de Geografía sin la utilización por el profesor y los educandos de un mapa para trabajar con él.

La Geografía es una asignatura que trabaja el primer nivel de abstracción; su objeto de estudio, el ecosistema "Hombre-Naturaleza", se produce en un espacio geográfico concreto y dentro del planeta Tierra, en el cual se evidencian las regularidades que en el proceso de esta relación se desarrollan y, por tanto, tienen que ser localizadas, y es objetivo fundamental de la asignatura hacerlo; de ahí que el trabajo con el mapa constituye objetivo esencial y método en el proceso de enseñanza-aprendizaje de la Geografía.

La Geografía debe seguir perfeccionándose y estudiándose dentro del currículo escolar cubano. Debe promover la reflexión y el diálogo de los encargados de su enseñanza para ajustarlo y hacerlo más representativo de la que se enseña en la escuela cubana en los inicios de este tercer milenio. Es una disciplina de gran valor educativo y formativo y, por último, debe plantearse problemas del espacio geográfico a los que puede dar solución a partir de su instrumentación metodológica.

No obstante, consideramos oportuno señalar que no en todos los casos los cambios y modificaciones introducidos en los programas de las asignaturas geográficas en la Educación Secundaria Básica durante los últimos cinco años han favorecido la enseñanza y el aprendizaje de la Geografía en la escuela.

Por otra parte, el nivel de preparación hoy de un considerable grupo de profesores en ejercicio no está en correspondencia con los programas que se han diseñado. De igual manera, se ha comprobado, a partir de la práctica, que de los matriculados en los cursos de postgrado sobre el trabajo con el mapa y otras representaciones cartográficas desarrollados por el autor, un número significativo de ellos demuestran dificultades con el dominio de los contenidos de esta disciplina.

En las fuentes consultadas de Didáctica de la Geografía y Metodología de la Enseñanza de la Geografía, publicadas tanto en Cuba como en el extranjero, para referirse a todo el proceder metodológico y didáctico con el mapa dentro y fuera de la clase se utilizan diferentes términos, entre los cuales se destaca: el trabajo con materiales cartográficos, el lenguaje cartográfico o de los mapas, el trabajo con las fuentes cartográficas y otros. Pero el término "trabajo con el mapa" es un término cubano, que aparece por primera vez definido 
en el año 1996 por el profesor Pedro Pablo Recio Molina, quien en el proceso de sistematización lo reconstruye en el año 2006 como parte de su tesis doctoral, como una concepción didáctica sin precisar su estructuración en la definición que ofrece.

Según este autor, el trabajo con el mapa debe ser considerado como una concepción didáctica, según se dijo anteriormente, en la que enfatiza que debe ser “...dirigida de manera intencional y sistémica a la estructuración de acciones con el mapa en el proceso de enseñanza-aprendizaje de la Geografía, para la codificación, decodificación y recodificación del contenido geográfico en él representado" (Recio Molina, 2006:33).

En la fase exploratoria de esta investigación, se sistematizaron los documentos orientados por el Ministerio de Educación en el período de 1970 al 1990, las tesis doctorales referidas al trabajo con el mapa, donde pudo constatar que existían documentos, circulares, orientaciones metodológicas y otros materiales en impresión ligera donde se expresaban orientaciones acerca del trabajo a desarrollar con el mapa en las escuelas. Es de destacar que en el libro Metodología de la Enseñanza de las Asignaturas Geográficas (Quesada Ginoris et al., (1988), al tratar los métodos de enseñanza-aprendizaje, se asume la clasificación binaria que tiene en cuenta la fuente de conocimientos: verbales, visuales y prácticos; y los niveles de independencia cognoscitiva a alcanzar por el estudiante: reproductivos, de búsqueda parcial e investigativos. El trabajo con el mapa lo expresan como un método dentro de la clasificación según la fuente de obtención del conocimiento.

En el Libro Metodología de la Geografía, de Francisco Materno, editado en 1976 y destinado a la formación del personal docente, al referirse al trabajo con el mapa se plantea que “(...) constituye una parte importante de la enseñanza de la Geografía” (Materno, 1976: 24) y señala tres aspectos de relevancia a tener en cuenta: conocimiento en el mapa del lugar del accidente o hecho geográfico estudiado, aptitud para indicar correctamente estos hechos y aptitud para leer el mapa, la que plantea en etapas, gradualmente, durante todo el curso de la asignatura, y no circunscrita a un grado en específico.

La investigadora Graciela Barraqué Nicolau, en los textos publicados $(1978,1991)$ revela que el trabajar con el mapa depende de la asimilación por el estudiante, de conceptos topográficos y cartográficos que le permiten conocer el mapa, lo que le posibilitará reflejarse en la conciencia el contorno de los territorios y las relaciones que entre ellos se establecen, para poder acceder a lo que se denomina lectura del mapa, donde deben ejercitar la interpretación de sus elementos para reflejar las características del territorio.

Esta misma autora incluye el trabajo con variados tipos de mapas como un método propio de la Geografía, criterio que no comparte totalmente este investigador, pues el trabajo con el mapa no es exclusivo de la Geografía, toda vez que otras ciencias que trabajan o investigan el espacio geográfico requieren de su utilización; ejemplo de ello son Historia, Biología, Climatología, Geomorfología, Biogeografía, entre otras.

Panchesnikova (1989), en su libro Metodología de la enseñanza de la Geografía, aborda el trabajo con el mapa como una vía para el desarrollo de la imaginación espacial, pues 
requiere el tránsito por tres etapas para su lectura: comprender el mapa, leerlo -momento en que destaca una lectura elemental y una lectura compleja- y, finalmente, conocer el mapa. De igual manera, destaca que en el proceso de enseñanza - aprendizaje se deben utilizar los métodos, según la actividad cognoscitiva del estudiante, clasificados por Lerner y N.N. Skatkin (1981) y que se aplican con la ayuda de las diferentes fuentes de los conocimientos geográficos.

La citada autora considera que “...el trabajo con estas fuentes forma los procedimientos de enseñanza en relación con este u otro método (...) así el trabajo con el mapa puede servirle como procedimiento a varios de los métodos de enseñanza-aprendizaje: el explicativo ilustrativo, reproductivo, exposición problémica, el parcial de búsqueda o el investigativo" (Panchesnikova, 1989: 57).

En el proceso de enseñanza-aprendizaje de la Geografía, el trabajo con el mapa posee sus propios procedimientos didácticos como método de enseñanza-aprendizaje de la Geografía.

Los procedimientos didácticos del trabajo con el mapa como método de enseñanza aprendizaje de la Geografía son:

○ Orientación adecuada por el profesor del objetivo expresado en el trabajo con el mapa.

- Selección adecuada de los mapas que se van emplear en la clase según el objetivo propuesto (mapas temáticos, atlas, mapas esquemas entre otros).

- Planteamiento de las tareas docentes que van a desarrollar los educandos en el trabajo con el mapa para cumplir el objetivo.

- Organización de la información del mapa y la representación matemática de los datos: título, orientación, la escala, leyenda, localización, distribución, distancia, superficie.

- Decodificación e interpretación de los símbolos empleados mediante la forma, tamaño y color.

- Explicación de la distribución geográfica. Para ello, determinación de las relaciones causa-efecto entre los objetos, fenómenos y procesos representados.

- Superposición de mapas y comparación de los fenómenos locales con los regionales, nacionales y mundiales (el uso de diferentes escalas).

- Contrastación de la información con otras fuentes (documentos escritos, libros de texto, enciclopedias, y otros.).

○ Comprensión, interpretación, comentario, análisis y valoración crítica de los diversos objetos, fenómenos y procesos geográficos.

- Comunicación de sus resultados derivados del trabajo con el mapa. 
- Aplicación a situaciones nuevas de lo aprendido con el trabajo con el mapa.

En las guías de estudio sobre Metodología de la Enseñanza de la Geografía, elaboradas en el año 1984 para los profesores en formación de los Institutos Superiores Pedagógicos, se destacaba el trabajo con mapas y con la esfera terrestre y lo clasifican dentro de las fuentes de obtención del conocimiento.

Otros autores, como Ginoris Quesada y otros (1988), ofrecen variadas ejemplificaciones de trabajo con el mapa y lo conciben solo como un procedimiento metodológico; Pérez Capote y otros (1991) tratan el trabajo con materiales cartográficos, en el cual evidentemente incluyen el mapa, declaran los niveles para su lectura y aseguran que esta debe ser lectura elemental, lectura compleja y lectura de superposición de mapas.

Canér Román (1996:24) ve el trabajo con el mapa como "una vía para el desarrollo de la creatividad”. Precisa las habilidades de trabajo con mapas que debe utilizar el estudiante en el proceso de enseñanza-aprendizaje de la Geografía y declara en sus publicaciones que es el método imprescindible en la Didáctica de la Geografía.

Recio Molina (2006), al accionar con el trabajo con el mapa, propone acciones y operaciones a realizar por los educandos para desarrollar la habilidad "Lectura del mapa" según los diferentes niveles de comprensión de la lectura, con un enfoque desarrollador en función de los niveles de lectura indicados como reproductivo, interpretación-aplicación y extrapolación. Todas estas concepciones de trabajo con el mapa quedan resumidas en el esquema que aparece en la figura siguiente:

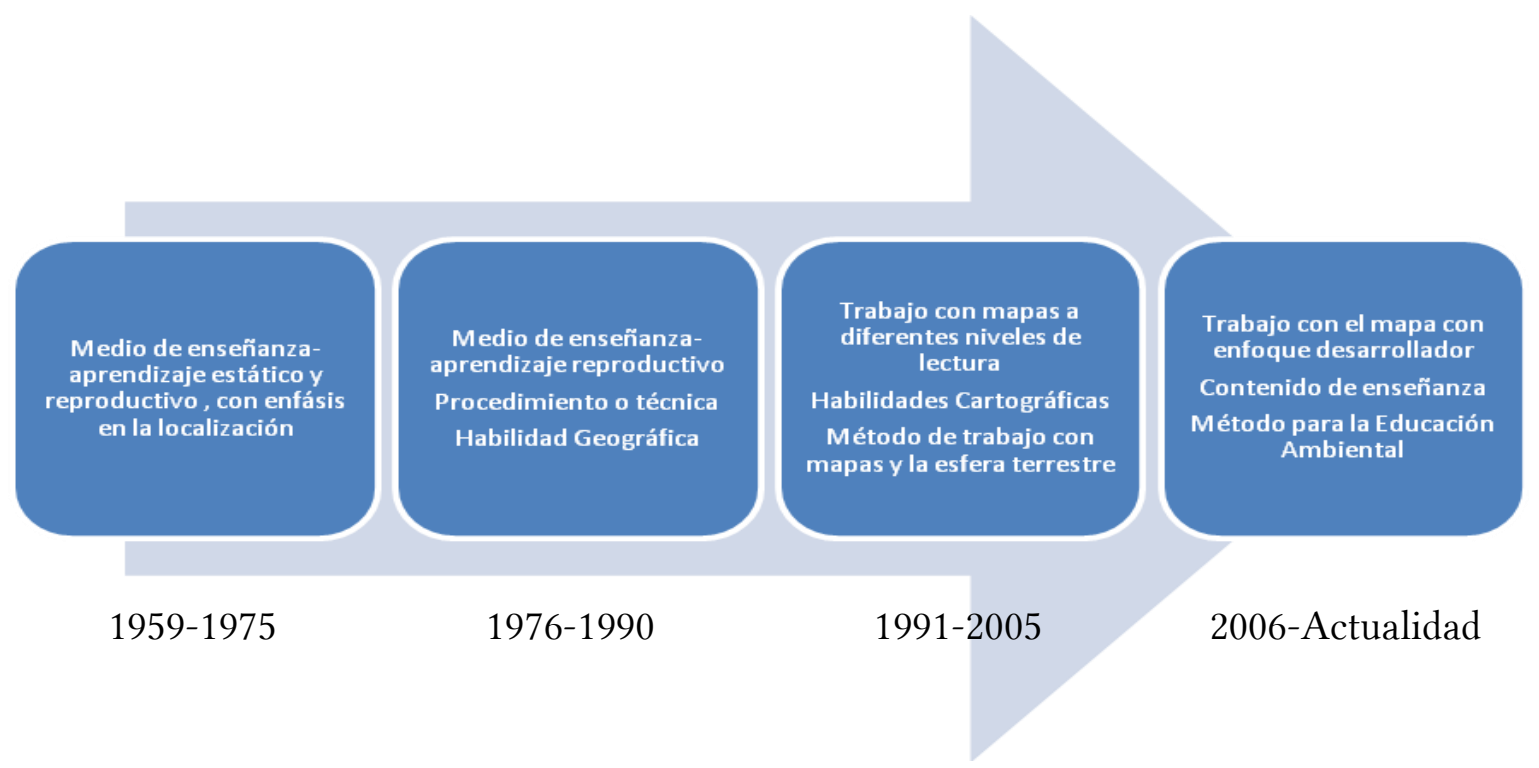

Figura 2. Concepciones de las actividades con el mapa en la enseñanza de la Geografía en Cuba. Fuente: Elaborada por el autor a partir de las fuentes consultadas en Didáctica de la Geografía o Metodología de la enseñanza de la Geografía. 
En resumen, se puede expresar que las diferentes concepciones de las actividades con el mapa en la enseñanza de la Geografía en Cuba le permitieron al autor precisar que en las dos primeras etapas no existía una concepción desde el trabajo con el mapa, la que fue abordada en el año 1985; por lo tanto, para el autor esas dos etapas le brindaron a la concepción didáctica que se propone el trabajo con el mapa como medio de enseñanza-aprendizaje en el proceso de enseñanza-aprendizaje de la Geografía y una concepción desde la empíria y que aparece reflejada en los textos de metodología donde se expresa que hay que desarrollar habilidades para el trabajo con el mapa; sin embargo, diferían entre si los autores. Por otra parte, el trabajo con el mapa constituía el método de la Geografía, pero sin una definición y una orientación al respecto.

Ya en la tercera etapa, desde el punto de vista teórico se precisan las habilidades, las acciones y operaciones de cada una de las invariantes para el trabajo con el mapa y se determina que para a todo proceder el trabajo con el mapa constituye una concepción didáctica

En la cuarta etapa se aplica esa concepción didáctica a la teoría del aprendizaje desarrollador donde se precisan las acciones del profesor y el estudiante para lograr el aprendizaje autorregulado, consiente, motivador y significativo.

Todos estos elementos se tuvieron en cuenta al definir que el trabajo con el mapa es un método de enseñanza de la Geografía.

En la escuela, en virtud de las transformaciones que se llevan a cabo, es necesario que se integren los conocimientos. La formación de profesores exige que se les prepare de manera integrada; sin embargo, la concepción de trabajo con el mapa actual carece aún de ese enfoque interdisciplinar que tiene por naturaleza. Un ejemplo que corrobora lo anterior es que en la asignatura Matemática, el estudiante debe desarrollar habilidades como trazar líneas, ángulos, medir y calcular, y otras. Sin embargo, en el trabajo con el mapa esto puede cumplirse para contribuir a incrementar el conocimiento geográfico y demostrar la aplicación práctica de los conocimientos matemáticos, y no se realiza regular y eficientemente.

Cuando los educandos en Biología estudian los hábitats de plantas y animales, rara vez se observa al profesor localizar en un mapa su ubicación espacial y, por tanto, la caracterización de ese hábitat pasa a ser una descripción reproductiva, cuando habría podido convertirse en demostración si se hubieran aplicado los conocimientos antecedentes de los educandos respecto del trabajo con el mapa.

En resumen, múltiples son los ejemplos que evidencian que a través del trabajo con el mapa en la escuela, este constituye una importante herramienta en la formación de una cultura general e integral del estudiante desde el proceso de enseñanza-aprendizaje de la Geografía, que puede utilizarse como método para la asimilación de los contenidos y como elemento de integración entre las asignaturas de la Educación Secundaria Básica, por lo que constituye un lenguaje que trasciende la disciplina Geografía escolar. 


\section{método de trabajo con el mapa}

En el proceso de enseñanza-aprendizaje de las diferentes disciplinas que se dan en la escuela, el componente método ocupa un destacado lugar, pues de él depende, en gran medida, el cumplimiento del objetivo y de los demás componentes de dicho proceso.

Etimológicamente la palabra método, procede del griego $\mu \varepsilon \theta o \delta o \varsigma$, con el significado de "camino a seguir", una vez dada la aspiración cognoscitiva, para su consecución como meta. Cobra el sentido ir en busca de... algo (un objeto, un problema...), de manera que se pueda encontrar, descubrir su lógica interna, es decir, conocer su esencia y sus relaciones esenciales.

En algunos de los casos, se ignora por parte de los profesores de Ciencias Naturales y de Geografía su importancia y se considera que el método de enseñanza-aprendizaje surge por el solo hecho del dominio del contenido. Por su parte, otros criterios sobrevaloran el papel del método, ignorando así la relación fundamental que existe entre todos los componentes del proceso de enseñanza-aprendizaje.

El método de enseñanza-aprendizaje constituye el componente del proceso de enseñanza-aprendizaje en el cual se ejecutan acciones y operaciones estructuradas de forma lógica y coherente por parte del profesor y el estudiante para la asimilación del contenido teórico y práctico de la enseñanza y así lograr el objetivo propuesto.

Es de señalar que no existe un método único o universal. Muchas son las posibilidades de combinarlos; es necesaria la conjugación de varios métodos para que el proceso de enseñanza-aprendizaje sea óptimo. Por otra parte, existen otros factores, como el número de educandos, las condiciones del aula, las habilidades y hábitos de trabajo precedentes, el desarrollo profesional del maestro y otros, que deben tenerse en cuenta en la aplicación de los métodos en las diferentes formas de organización del proceso de enseñanza-aprendizaje.

El mapa geográfico como medio de enseñanza-aprendizaje de la Geografía es reconocido por la Didáctica General, por la Didáctica de la Geografía y por los autores. Sin embargo, el mapa por sí solo no es un simple medio de enseñanza, instrumento didáctico o pura ilustración icónica del contenido representado; es parte de él y debe ser utilizado como tal para la comprensión de ese contenido. El trabajo con el mapa es el resultado de una selección de acciones y operaciones y debe ser entendido por el estudiante para interpretar, comprender y valorar adecuadamente la información cartográfica que contiene. De ahí que se le considere como un método de enseñanza-aprendizaje.

"El trabajo con el mapa es la base primordial de la enseñanza de la Geografía" (Barraqué Nicolau, 1991), es el método esencial y más representativo de la Geografía porque es el que la distingue de otras ciencias; es fundamental para la comprensión compleja del espacio geográfico que se manifiesta en la multiplicidad de relaciones establecidas entre la 
sociedad y la naturaleza. Se puede plantear que es imposible enseñar Geografía en la escuela sin la presencia del trabajo con los mapas.

El trabajo con el mapa es un método de enseñanza-aprendizaje de la Geografía, ya que es capaz de conducir al logro del objetivo planteado en el proceso de enseñanza-aprendizaje al identificar y caracterizar los contenidos de la Geografía y a su vez formar y sistematizar dentro de estos, los conocimientos y habilidades (intelectuales y prácticas) propios de esta disciplina en los educandos, a partir de una orientación lógica y coherente de acciones y operaciones de carácter reproductivo y productivo con el mapa para el procesamiento de la información representada.

En este sentido, el trabajo con mapas posee elementos que lo caracterizan como método para la enseñanza-aprendizaje de la Geografía, ya que constituye:

○ Objetivo esencial en los programas de todos los niveles de enseñanza.

- Una vía mediante la cual se adquieren conocimientos, hábitos, habilidades, capacidades y valores que requieren de la ejecución lógica y coherente de un sistema de acciones y operaciones a realizar por profesores y educandos.

- Un medio en el que está representado el contenido de enseñanza en un lenguaje muy peculiar, el "cartográfico", asimilado por el estudiante a partir del sistema de habilidades intelectuales y propias de trabajo con el mapa.

El trabajo con el mapa es método de enseñanza-aprendizaje porque en su carácter dinamizador del proceso de enseñanza-aprendizaje de la Geografía está en función del objetivo a cumplir por el profesor y el estudiante en la clase, por la estrecha relación que establece con el resto de los componentes del proceso de enseñanza-aprendizaje, por el sistema de acciones y operaciones que de forma lógica y coherente desarrollan los profesores y educandos en la asimilación de los contenidos representados en los mapas que trabaja en la clase. Además, por su dinámica expresada en su aspecto externo e interno, en lo lógico y psicológico, en correspondencia con las funciones didácticas que se desarrollan dentro del proceso de enseñanza-aprendizaje de la Geografía.

El trabajo con el mapa es método de enseñanza-aprendizaje porque incrementa el interés del estudiante en su desempeño con él como medio en Geografía y en aquellas asignaturas que analicen manifestaciones espaciales de distintos fenómenos. Es capaz de romper con lo tradicional que hoy se encuentra en las escuelas, de superar la clase memorística, descriptiva, alejada de la realidad, carente de interés, en la cual el protagonista sea el estudiante y no el profesor.

El trabajo con el mapa es método de enseñanza-aprendizaje porque posibilita establecer la correspondencia necesaria entre los conceptos adquiridos por los educandos y los que se encuentran representados en este; también contribuye al desarrollo de la independencia cognoscitiva, a la estimulación de diferentes componentes de la capacidad creadora y a la comunicación entre ellos. 
Por otra parte, tiene otras razones que lo identifican como método para la enseñanza de la Geografía. Dentro de ellas se destaca:

- A través del trabajo con el mapa (aunque no solo por ello) se puede tener una conceptualización más exacta del espacio geográfico, que es, junto con el tiempo, uno de los dos ejes estructurantes que sirven para la comprensión del mundo en que desarrolla el estudiante.

- Los procedimientos del trabajo con el mapa que se desarrollan en la clase presentan características propias que los distinguen de otros procedimientos de tratamiento de la información.

- El trabajo con el mapa facilita de manera importante la consecución de algunos de los objetivos generales que se atribuyen a las asignaturas del área de Ciencias Sociales y Naturales y que tienen que ver con la comprensión del espacio geográfico, como por ejemplo, localizar los objetos, fenómenos y procesos, explicar sus distribuciones, conocer y comprender problemas ligados a la utilización de los recursos naturales por las sociedades

- Tiene utilidad práctica, dadas las múltiples situaciones cotidianas que se nos presentan (mapas de carreteras, planos de localidades, catastro).

- Hay conceptos geográficos que desarrollados en el trabajo con el mapa se aprenden mejor.

○ Constituye un instrumento de análisis y generalización.

- Por ser un lenguaje visual permite una aprehensión global de fenómenos concretos y abstractos, estáticos y dinámicos, actuales, pasados y futuros.

- Está presente en todas las etapas del método científico y resulta un método idóneo para plasmar resultados alcanzados en cualquier investigación, ya sea de carácter geográfico, social, histórico u otras.

Por otra parte, el método de trabajo con el mapa se caracteriza por requerir diferentes niveles de complejidad, en correspondencia con la base de conocimientos y el sistema de acciones y operaciones que se ejecutan. Recio Molina (2006) destaca tres niveles fundamentales: un nivel reproductivo, un nivel de interpretación y un nivel de aplicación, todos encaminados a alcanzar un resultado desde el punto de vista instructivo, educativo y desarrollador.

Resulta significativo, como se planteó anteriormente, que el trabajo con el mapa no es solo atributo de las asignaturas geográficas. Otras ciencias y asignaturas particulares requieren de su utilización ya que presenta un lenguaje que trasciende a la geografía misma, pues su ubicación espacial no es privativa de hechos de tipo físico y económico. Es importante analizar cómo la Geomorfología, la Meteorología, la Cartografía, la Historia, la Bio- 
logía y otras ciencias lo utilizan, ya sea como fuente de conocimiento o para la investigación. Pero si el trabajo con los mapas se utiliza para localizar los objetos, fenómenos y procesos que ocurren en un determinado espacio geográfico y para darles una explicación, un significado; para buscar la conexión y su variación espacio-temporal desde el punto de vista de la Geografía, entonces se puede plantear que es un método propio de ella.

El trabajo con el mapa como método tiene la importante misión de alfabetizar cartográficamente al estudiante a partir de la lectura e interpretación de sus códigos, lo que permite una mejor comprensión del ecosistema” Hombre - Tierra” en el espacio geográfico concreto; por otra, parte trasmite, comunica una realidad que permite al estudiante interpretarlo de manera crítica, transformadora y cooperativa, con lo que logra el desarrollo de sus capacidades cognitivas, procedimentales y axiológicas. "La educación se produce en un medio concreto, en un contexto geográfico, natural, social y cultural que proporciona estímulos, valores, actitudes, conductas y, en definitiva, contenidos educativos" (Jérez García, 2011:28).

\section{CONCLUSIONES}

El trabajo con el mapa como método de la Geografía constituye una valoración teórica imprescindible dentro de la Didáctica de la Geografía, por el aporte teórico que presenta y que debe ser expresado desde una concepción que revolucione la manera de darle tratamiento de los contenidos geográficos desde el proceso de enseñanza-aprendizaje de la Geografía mediante el trabajo con el mapa.

El trabajo con el mapa es el método representativo de la Geografía, ya que permite desde la lectura cartográfica y sus procedimientos el reconocimiento, interpretación, comprensión y valoración de los objetos, fenómenos y procesos geográficos.

\section{REFERENCIAS}

ADDINE FERNÁNDEZ, F. (2007). Didáctica: teoría y práctica. La Habana: Editorial Pueblo y Educación.

BARRAQUÉ NICOLAU, G. (1991). Metodología de la enseñanza de la Geografía. Ciudad de La Habana: Editorial Pueblo y Educación.

BÁYEUX, F. (1999). Tratamiento Metodológico de las Habilidades Cartográficas en séptimo grado. [Tesis de Doctorado]. La Habana Cuba

CANER ROMÁN, A. (1996). La creatividad en el trabajo con mapas. [Tesis de Maestría] ISPEJV. Ciudad de La Habana. 
CUÉTARA LÓPEZ, R. (2004). Hacia una Didáctica de la Geografía Local. La Habana: Editorial Pueblo y Educación.

DURÁN, D. (2009). El valor formativo de la Geografía. Consultado el 24 de marzo de 2011, de http://geoperspestivasblogspot.com

FERIA ÁVILA, H. (2004). Propuesta de superación profesional de posgrado especializada para elevar el dominio del profesor de Geografía en relación con las habilidades cartográficas docentes. [Tesis de Doctorado]. Las Tunas.

GÓMEZ TORRES, J. (2014). Concepción didáctica de trabajo con el mapa geográfico como método para el desarrollo de la educación ambiental en la Secundaria Básica. Revista Órbita Científica. No 78. vol. 20.

JEREZ GARCÍA, Ó. (2011). El lenguaje cartográfico como instrumento para la enseñanza de una geografía crítica y para la educación ambiental. Madrid: Universidad Complutense de Madrid.

LAGUNA CRUZ, J A. (2004). Concepción didáctica para el desarrollo de habilidades para la enseñanza- aprendizaje de los contenidos geográficos empleando materiales cartográficos. Revista: Entorno Geográfico. Holguín: Universidad de Ciencias Pedagógica "José de la Luz y Caballero".

MATERNO, F. (1976). Metodología de la Geografía. Ciudad de La Habana: Editorial Pueblo y Educación.

MINISTERIO DE EDUCACIÓN, Cuba. (1985). El plan de perfeccionamiento y desarrollo del Sistema Nacional de Educación de Cuba. Dirección y Producción de Medios de Enseñanza, La Habana: Editorial Libros para la Educación.

PANCHESNIKOVA, L.M. (1989). Metodología de la Enseñanza de la Geografía. La Habana: Editorial Pueblo y Educación.

PÉREZ CAPOTE, M. (2002). Didáctica de la Geografía. Selección de Temas. Ciudad de La Habana: Editorial Pueblo y Educación.

PÉREZ CAPOTE, M. (2000). Los métodos de enseñanza de la Geografía: una encrucijada para el desarrollo de la actividad cognoscitiva de los estudiantes (en soporte digital), La Habana: Editorial Pueblo y Educación.

RECIO MOLINA, P.P. (2006). Estrategia didáctica de trabajo con el mapa con enfoque desarrollador en el proceso de enseñanza-aprendizaje de la Geografía en la educación Secundaria Básica. [Tesis de Doctorado] La Habana: UCP "Enrique José Varona". 